\title{
Incidence of venous thromboembolism in japanese trauma population
}

\author{
R Miki , S Kikuta, T Koga, S Kai, A Inoue, T Kawase, S Ishihara, S Nakayama \\ From ESICM LIVES 2015 \\ Berlin, Germany. 3-7 October 2015
}

\section{Introduction}

There have been several manuscripts reporting Asians having lower risk for developing venous thromboembolism (VTE) in the medical population. However, incidence of VTE in the Asian trauma population is not fully known.

\section{Objectives}

To investigate the incidence of deep venous thrombosis (DVT) and massive or submassive pulmonary embolism $(\mathrm{PE})$ in Japanese trauma population.

\section{Methods}

This single center observational study was conducted at tertiary emergency and critical care center in Japan. From April 2009 to March 2015, all trauma patients admitted to intensive care unit (ICU) were enrolled in this study. To investigate the incidence of DVT, all patients were risk stratified according to VTE risk assessment checklist on admission and adequate thromboprophylaxis was performed. Patients were screened for DVT by lower extremity duplex ultrasonography (US) six-days after admission and the incidence of DVT was assessed. DVT in popliteal vein or above was defined proximal and others were defined distal. To investigate the incidence of PE, all of the computed tomography $(\mathrm{CT})$ reports were searched retrospectively by keyword "PE" or "VTE" during the study period including autopsy imaging. Massive and submassive PE was defined by both presence of thrombi in the main pulmonary artery and right ventricular diameter greater than that of left ventricle by contrast enhanced CT exam, suggesting of right ventricular pressure load. Patients who had PE by contrast enhanced CT either by coincidence or due to clinical symptom were identified.

Hyogo Emergency Medical Center, Emergency Medicine and Critical Care, Kobe, Japan

(c) 2015 Miki et al.; This is an Open Access article distributed under the terms of the Creative Commons Attribution License (http:// creativecommons.org/licenses/by/4.0), which permits unrestricted use, distribution, and reproduction in any medium, provided the original work is properly cited.

\section{Results}

During the six-year study period, 2,618 trauma patients were enrolled. Mean age was 48.7 years and 1,885 patients (72\%) were male. Blunt injury accounted for 2,339 patients (89\%), and mean injury severity score was 20 . Blood products were transfused in 572 patients $(22 \%)$ and 1,144 (44\%) required operation or interventional radiology within 24 hours of arrival. In-hospital mortality was $13 \%$. By using duplex US, 916 patients were screened for DVT. Of those 916 patients, 29 patients (3\%) developed proximal DVT and 202 patients (22\%) developed distal DVT during ICU stay. Among 2,618 trauma patients, 2 patients $(0.2 \%)$ developed massive or submassive PE during ICU stay, of which one died due to PE. There were 10 cases $(0.4 \%)$ of non-massive or non-submassive PE identified by contrast enhanced CT as well.

\section{Conclusions}

The incidence of proximal and distal DVT in Japanese trauma population admitted to ICU was $3 \%$ and $22 \%$ respectively. The incidence of massive or submassive PE was $0.2 \%$. A larger study is warranted to reveal an accurate risk of VTE and determine optimal anti-coagulation measure in Asian trauma population, considering low incidence of VTE in this study.

\section{Published: 1 October 2015}

\section{References}

1. White $\mathrm{RH}$, et al: The Epidemiology of Venous Thromboembolism. Circulation 2003, 107:-1-4-I-8.

2. Barrera $L M$, et al: Thromboprophylaxis for trauma patients. Cochrane Database Syst Rev 2013, 3:CD008303, Mar 28.

doi:10.1186/2197-425X-3-S1-A378

Cite this article as: Miki et al:: Incidence of venous thromboembolism in japanese trauma population. Intensive Care Medicine Experimental 2015 3(Suppl 1):A378. 